\title{
Superficial Peroneal Nerve
}

National Cancer Institute

\section{Source}

National Cancer Institute. Superficial Peroneal Nerve. NCI Thesaurus. Code C92603.

A branch of the common peroneal nerve. It innervates the surface of the calf and foot. 